\title{
URBAN SUSTAINABLE ECOSYSTEMS ASSESSMENT THROUGH AIRBORNE EARTH OBSERVATION: LESSONS LEARNED
}

\author{
R. Alamús*, F. Pérez, L. Pipia, J. Corbera. \\ Institut Cartogràfic i Geològic de Catalunya, Parc de Montjuïc 08038 Barcelona, Spain \\ (ramon.alamus, fernando.perez, luca.pipia, jordi.corbera)@icgc.cat
}

Commission I, WG I/1 \& WG I/6

KEY WORDS: Multi-Sensor, Urban, Light Pollution, UHI, Hyperspectral Sensor, Thermal Sensor, Airborne Remote Sensing

\begin{abstract}
:
Europe's big cities and metropolitan areas constitute an ecosystem where climate trends are having, and will continue to have, a big impact on the population in the short, medium, and long term. Therefore, the task of generating a mapping approach over urban ecosystems to provide indicators assessing their sustainability and facilitating the design and implementation of policies and derived actions has become a priority for the Institut Cartogràfic i Geològic de Catalunya (ICGC) as a mapping agency.

The ICGC has been using its own assets and competences in airborne Earth Observation for data capture and processing as well as inclusion of additional data such as cadastral databases and digital surface models to generate a set of urban sustainability indicators. ICGC has designed flight campaigns at a very high spatial and spectral resolution in visible, near infrared and long wave infrared regions, processed geospatial data and finally transformed geoinformation into demanded ICGC products over urban ecosystems such as urban green, solar potential, light pollution, energy leaks or urban heat island effect. This approach has been applied in coordination with urban planning stakeholders in different cities and is planned to be extended to the majority of urban ecosystems in Catalonia.
\end{abstract}

This paper presents a critical summary of the main advantages, disadvantages, constrains and lessons learned in the search for feasible, available and desirable responses from the earth-observation airborne-sensor point of view to the main urban ecosystem challenges.

\section{INTRODUCTION}

Earth observation systems offer a repetitive, synoptic and therefore privileged view of the territory. The advantages that a platform at a certain height can offer have been exploited for more than a century. In more recent times airborne capabilities have been enlarged with the operational use of satellite and unmanned airborne platforms. Nowadays, the wide range of spectral, spatial and temporal resolutions provided by Earth observation platforms represent a challenge of use and applicability that runs from optical, thermal or active systems, such as Lidar systems or radar, and drive us to a better knowledge of the territory, thus, a better management of its resources, impacts, affections and changes, transforming Earth observation data into decision -support instruments.

The indicators of urban sustainability are tools that must be enable urban managers and decision makers to measure socioeconomic and environmental impacts as well as facilitate the diagnosis of problems and potential actions to undertake. The observation of the Earth, from data capture to transformation into geoinformation, represents a spatial and temporal approximation of measurement of these indicators.

ICGC, with its airplanes, sensors and competences to transform data into knowledge and wisdom, is deeply involved to solve the usability challenge on Earth observation data, particularly in urban ecosystems from the perspective of providing indicators and knowledge in the search for a better design and sustainable management of urban ecosystems.
For the management of urban and peri-urban ecosystems, it is essential to have specific information related to anthropic and environmental variables. This paper is focused on the use and lessons learned dealing with hyperspectral and photogrammetric sensors of the ICGC over urban ecosystems in visible-near infrared (VisNIR) and long wave infrared (LWIR) spectral ranges.

\section{SENSORS AND DATA CAPTURE}

The products committed to ICGC by urban stakeholders require a wide variety of sensors and methodologies from the data acquisition to the fainal product generation. A subsection is dedicated to describe sensors and methods for each of the topics under study.

\subsection{Light pollution: luminance and light sources}

ICGC sensors involved in the analysis/mapping of light pollution are an AISA Eagle II and a DMC-I photogrammetric frame camera.

The AISA Eagle II is a hyperspectral VisNIR pushbroom imager with a reflection grating and a two-dimensional CCD (charge coupled device) solid-state array detector, manufactured by SPECIM. The instrument operates by looking down in a fixed direction and imaging successive lines of the flown scene, building up a two-dimensional image as the platform moves forward (main parameters are described in table 1). One dimension of the CCD covers then the acrosstrack spatial direction (configurable in 512 or 1024 spatial pixels); the other one accounts for the spectral domain 
(configurable up to 256 bands covering the spectral range 400$1000 \mathrm{~nm})$

DMC-I is a high-resolution photogrammetric frame camera, manufactured by Z/I imaging (currently Hexagon) (Dörstel, 2003), which simultaneously captures one high-resolution (HR) panchromatic and four low-resolution (LR) multi-spectral (red, green, blue and near-infrared) images (main parameters are described in table 2). The across-track and along-track ratio between multispectral and panchromatic imaging is $1: 4$. The high resolution image is the result of mosaicking four subimages acquired by four slightly inclined panchromatic camera heads. Each of them covers approximately a quarter of the final image, called virtual image. The four low resolution multispectral images in the red, green, blue and near-infrared bands are acquired with four additional nadir-looking camera heads with a focal length of $25 \mathrm{~mm}$. Note that the four images completely cover the virtual high resolution image.

\begin{tabular}{|l|c|c|}
\hline Parameters & AISA Eagle II & DMC-I \\
\hline Sensor geometry & Pushbroom & Frame \\
FOV[] & 37.7 & $69.9 \times 42$ \\
\# pixels & 1024 & $13824 \times 7680$ \\
\# spectral bands & Up to 256 & 1 panHR / \\
& & 4 (RGBNir)LR \\
Spectral range [nm] & $400-1000$ & $400-1000$ \\
Focal length[mm] & 17.8 & 120 panHR / \\
& & $25 \mathrm{LR}$ \\
GSD @ 2500 m [m] & 1.5 & 0.25 \\
\hline
\end{tabular}

Table 1. AISA Eagle II and DMC-I main parameters.

All data are acquired in a single nocturnal flight where both sensors (AISA and DMC) are operated simultaneously in an airborne platform, which is able to carry both sensors. As the goal is the analysis of artificial nocturnal lights, it is required to avoid natural light sources, mainly moon light. Night flights must be ideally performed close to the New Moon phase when moon has already set.

Moreover, due to safety reasons related to aerial control authorities, nocturnal flights may have some operational restrictions, which depend on capabilities of the airplane, terrain and distance to the nearest airport. Such restrictions may limit the resolution of the resulting luminance map. Table 2 shows standard flight parameters for light pollution projects.

\begin{tabular}{|l|c|c|}
\hline & AISA Eagle II & DMC-I \\
\hline Flight season & \multicolumn{2}{|c|}{ Winter } \\
Flight time & \multicolumn{2}{|c|}{$20: 00-22: 00$} \\
Height AGL [m] & \multicolumn{2}{|c|}{$2500-3000$} \\
GSD [m] & $1.5-1.85$ & $0.25-0.30$ \\
Side lap [\%] & 40 & 70 \\
End lap [\%] & - & 70 \\
\hline
\end{tabular}

Table 2. Standard light pollution flight parameters

\subsection{Thermal behaviour: UHI and temperature gradient}

Sensor involved in the analysis/mapping the UHI and the thermal gradient is a TASI sensor.

The TASI is a hyperspectral LWIR pushbroom imager, with a dispersing prism and a two-dimensional MCT (MercuryCadmium-Telluride) long infrared array detector, manufactured by ITRES. The instrument operates by looking down in a fixed direction and imaging successive lines of the flown scene, building up a two-dimensional image as the platform moves forward (main parameters are described in table 3). One dimension of the detector covers the across-track spatial direction (600 spatial pixels); the other one accounts for the spectral domain (32 bands covering the spectral range 8-11.5 $\mu \mathrm{m})$.

\begin{tabular}{|l|c|}
\hline Parameters & TASI \\
\hline Sensor geometry & Pushbroom \\
FOV $\left.^{\circ}\right]^{-}$ & 40 \\
\# pixels & 600 \\
\# spectral bands & 32 \\
Spectral range $[\mu \mathrm{m}]$ & $8-11.5$ \\
Focal length[mm] & 25 \\
GSD@2500 m $[\mathrm{m}]$ & 3.0 \\
\hline
\end{tabular}

Table 3. TASI main parameters

Data is acquired in a double night-time flight, preferably in winter time. As the goal is the analysis of anthropogenic heat (for both topics under evaluation, UHI analysis and thermal gradient analysis), it is desirable to avoid additional heat source contributions, mainly the effects of shadows and temperature fluctuations of surfaces under sunlight and traffic flow. The UHI effect is most pronounced at night (when urban areas remain warm due to high heat absorption during the day, while rural areas rapidly cool off) and in winter (when temperature gradient between urban and rural areas is larger) (Harris and Coutts, 2011).

As in the case of light-pollution flights, night-flight restrictions may limit the resolution of the resulting temperature map. Table 4 shows standard flight parameters for thermal behaviour analysis projects.

\begin{tabular}{|c|c|}
\hline & TASI \\
\hline Flight season & Winter \\
Flight time (flight 1) & $00: 00$ \\
Flight time (flight 2) & $06: 00$ \\
Height AGL [m] & $1700-3000$ \\
GSD [m] & $2.0-3.6$ \\
Side lap [\%] & 40 \\
\hline
\end{tabular}

Table 4. Standard thermal behaviour flight parameters

\subsection{Solar Potential}

Sensors involved in the analysis of the solar potential are Lidar and, if necessary, a photogrammetric camera.

Data are acquired in diurnal flights. As the goal is the analysis of the potential performance of solar panels on rooftops, it is required to detect roof planes. Then Lidar data is acquired at a minimum density of 4 points $/ \mathrm{m}^{2}$.

\subsection{Urban Green}

Sensor involved in the urban green mapping is the DMC-I photogrammetric frame camera.

DMC-I is a high-resolution photogrammetric frame camera already described in the section 2.1, its main parameters described in table 1 .

Data are acquired in a diurnal flight. As the goal is the quantification of vegetation health/vigour in the urban environment, the interest is focused on the multispectral capabilities of the photogrammetric camera. Table 5 shows standard flight parameters for urban green projects. 


\begin{tabular}{|l|c|}
\hline & DMC-I \\
\hline Flight epoch & - \\
Flight time & $12: 00$ \\
Height AGL [m] & 900 \\
GSD HR [cm] & 9 \\
GSD LR [cm] & 36 \\
Side lap [\%] & 70 \\
End lap [\%] & $60-70$ \\
\hline
\end{tabular}

Table 5. Standard urban green flight parameters

\section{METHODS AND DERIVATED PRODUCTS}

\subsection{Light pollution: luminance map}

The high resolution luminance map creation is composed of two main steps: I) a low-resolution luminance map is retrieved from the hyperspectral sensor, and ii) the high resolution panchromatic ortho-image, derived from the photogrammetric camera, is intercalibrated with the low-resolution luminance map to enhance its spatial resolution.

At operational night flights, hyperspectral VisNIR sensor is set up to collect 126 bands of the radiation in $400-900 \mathrm{~nm}$ spectral range emitted or reflected by the surface. These bands are converted to luminance values at ground level and then combined to retrieve the visual perception of human eye. The spectral sensitivity of the human visual system is described by the photopic luminosity function which indicates the sensitivity of human eye to incoming light radiation at different wavelengths (CIE 1931). The conversion from measured radiance to luminance at ground level is performed in several steps. First, a radiometric calibration converts digital numbers captured by VisNIR sensor into radiances at flying height. These radiances are converted into radiances at ground level by compensating for the atmospheric attenuation and then the 126 band radiances are combined using the photopic luminosity function. Finally, a low resolution luminance map (at a resolution of $1.5 \mathrm{~m}$ GSD) for the entire area is created by mosaicking the imagery from the different flight tracks of the hyperspectral sensor. Figure 1 shows a detail of the low resolution luminance map segmented into 10 classes from 0.35 to $10 \mathrm{~cd} / \mathrm{m}^{2}$.

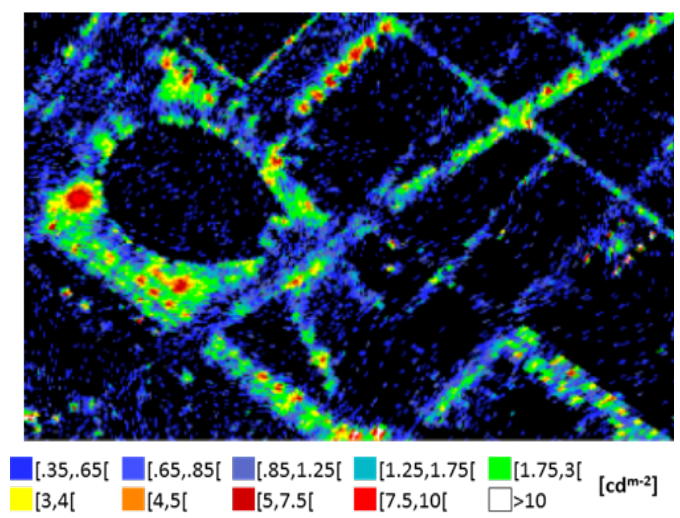

Figure 1. Low resolution map derived from Hyperspectral sensor. Segmentation in 10 classes

The dynamic range of the DMC panchromatic band is higher than that of the VisNIR sensor since the DMC spectral bands are much broader and, consequently, more photons can reach the charge-coupled device (CCD) in the image plane. The fusion consists of fitting the radiance values of the DMC images to the luminance map retrieved from the hyperspectral sensor. Once this calibration process has been completed, the luminance map at $0.25 \mathrm{~m}$ GSD can be computed from DMC imagery alone. Figure 2 shows a detail (same area as in figure 1) of the high resolution luminance map segmented into 10 classes from 0.35 to $10 \mathrm{~cd} / \mathrm{m}^{2}$.

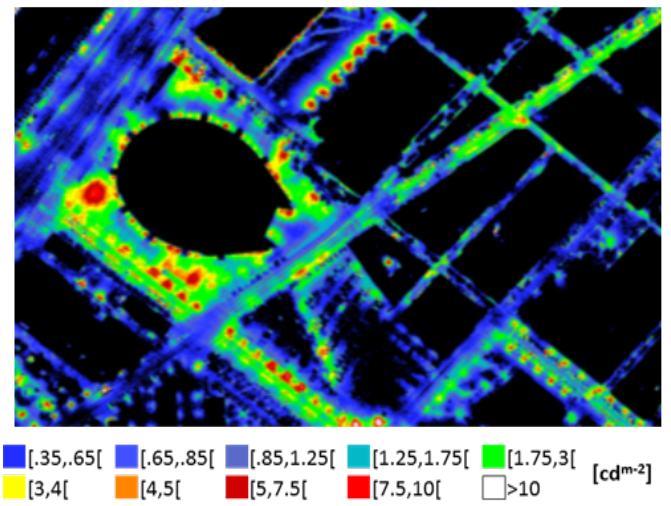

Figure 2. High resolution luminance map derived from the hyperspectral sensor and photogrammetric camera fusion. Segmentation in 10 classes

Further explanations may be found in (Pipia et al. 2014) and (Alamús et al. 2017).

\subsection{Light pollution; light source type map}

As shown in figure 3, artificial light sources have specific spectral signatures. The hyperspectral capabilities of the AISA Eagle II sensor allow deriving a set of spectral indexes focusing in particular features of the light spectral signatures, which are used to discriminate them.

The hyperspectral instrument AISA Eagle II has been designed for recording reflected sunlight and not for capturing artificial light at night. Then, the relatively low intensity of artificial light at night and the limited exposure time by operational restrictions causes hyperspectral images to be affected by high noise level.

In order to overcome this handicap a two-step strategy has been designed. First, spot source lights and their illuminated neighborhood are detected in the DMC nocturnal images. After that, a set of light spectral indexes is computed as a weighted average of spectral indexes extracted from the AISA hyperspectral data.

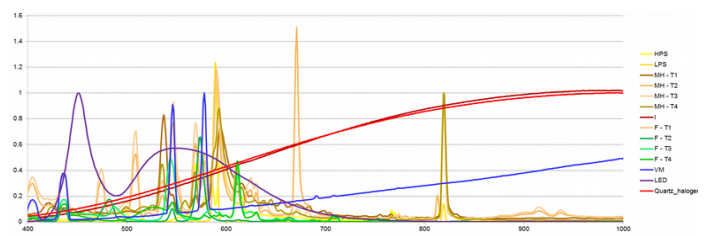

Figure 3. Spectral signature of different types of artificial light sources. (Units on $\mathrm{x}$-axis are nanometers)

Spot source lights are detected as the point (pixel) with the highest light intensity in its neighborhood. In a first step, for each image pixel the pixel with a maximum light intensity is detected in a $6 \mathrm{~m}$ radius neighborhood. Finally, for each one of these pixels, its neighborhood is grown up to the area that is mainly illuminated by the spot light source. Such point-like source describes the position where light intensity is the highest in its neighborhood, and is likely to correspond to the lamp center. 
Figure 4 shows a detail of detected spot source lights (greyblue dots) and their illuminated neighborhood (grey polygons). The theoretical locations of public lamps (colored circles) are superimposed on the image. Notice that most of the public lamps are close to a detected punctual source light. Nevertheless, in low light intensity area there are source lights that correspond to vehicles or reflective surfaces (for example, ground painted lanes, metal surfaces...) instead of public lamps.

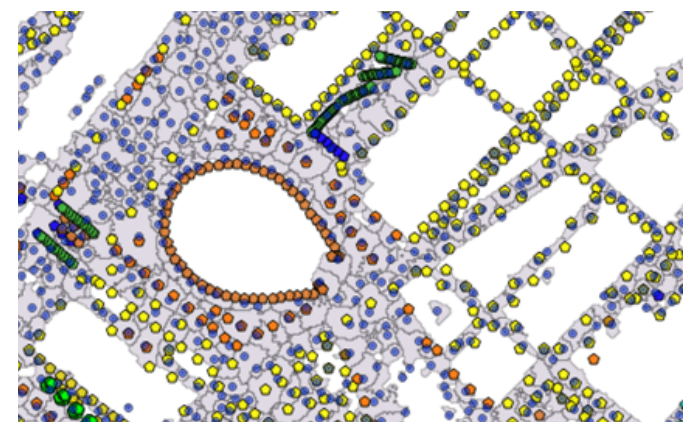

Figure 4. Detection of spot source lights and their illuminated neighbourhood.

Taking advantage of the different spectral signatures of the light sources (fig 3), a set of indexes has been derived. This set of indexes is used to derive the main type of the light source in the neighbourhood of the source light. Notice that each neighbourhood should be mainly influenced by the principal light source: the maximum calculated before. However, this neighbourhood may be also influenced by other light sources such as car lamps or showcase lights and light source type will be also influenced by the reflectivity features of the imaged surface. Figure 5 shows a detail of the resulting light source type map. The classification categories are the following (the main ones in our urban ecosystems): high pressure sodium lamps (yellow), metal-halide lamps (orange), mercury vapour lamps (blue), fluorescent lights (green), LED lamps (violet), halogen or incandescent lamps (red) and unclassified lamps (grey).

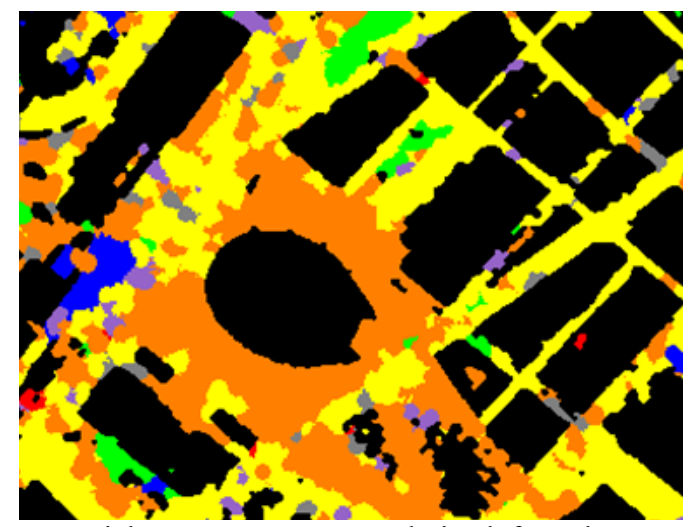

Figure 5. Light source type map derived from hyperspectral sensor.

\subsection{Thermal behavioiur; UHI}

The UHI map is derived from the data acquired during the close-to-midnight.

As the hyperspectral LWIR sensor collects 32 bands of the radiation in $8-11.5 \mu \mathrm{m}$ spectral range emitted by the surface, a geocoded temperature image is retrieved from each flight track using the NCEP atmospheric profile (Barsi et al. 2003) fitted to the ground information provided by the closest automatic weather station in the METEOCAT network (http://en.meteocat.gencat.cat/xema?lang=en), and a version of the TES technique tailored to TASI spectral properties (Pipia et al. 2013).

Concerning UHI analysis, the topic of interest is the air temperature at street level, thus a true orthoimage of the temperature image is derived (at a GSD around $2 \mathrm{~m}$ according to flight conditions), in order to discriminate between rooftops and street. This way, buildings can be masked out.

UHI phenomena is related to the temperature gradient (due to anthropogenic factors) between central, densely urbanized sectors and rural areas nearby. A set of rural areas free of forest, buildings or greenhouses is selected in order to evaluate the average temperature in rural areas. This average temperature becomes the average rural reference temperature. Then UHI map is computed as the subtraction of the rural reference temperature to the previously computed temperature orthoimage. Figure 6 shows an UHI image sample where buildings footprints are in transparency, UHI image is the gradient respect to the reference temperature (represented as zero) and the isothermal contour lines are plotted every $0.5^{\circ}$ increment.

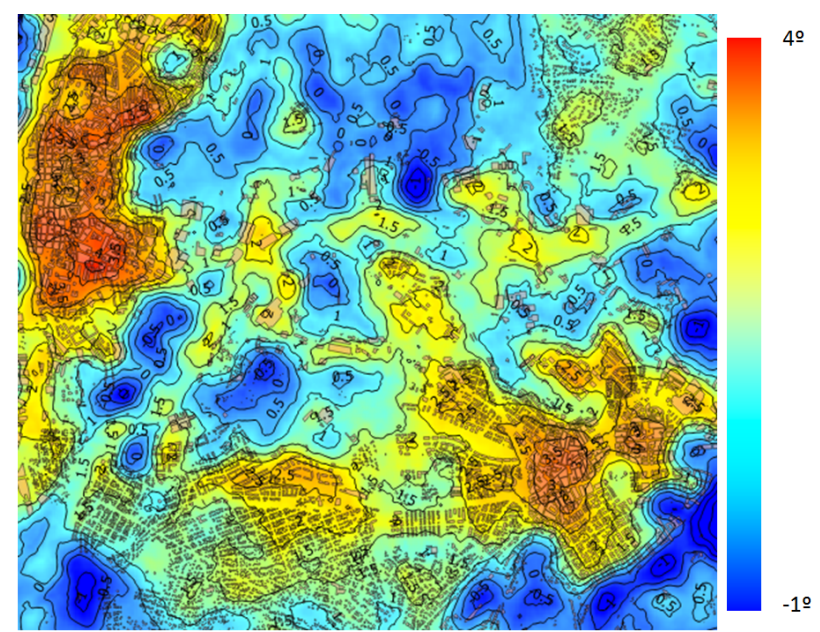

Figure 6. UHI map sample. Building footprints are shown in transparency, isothermal contour lines are drawn in black every $0.5^{\circ}$

\subsection{Thermal behavioiur; temporal temperature gradient}

The TASI allows measuring and retrieving information concerning the accurate temperature of the surface and emissivity spectrum of the imaged scene. Then, TASI-based absolute temperature images are reliable descriptors of possible heat leaks through roof covers when it is flown over man-made areas as urban areas and industrial districts. When there are two flights available it is possible to detect changes in temperature patterns.

Both flights, at 0.00 and at 6.00 , are processed to derive thermal maps as in the case of the UHI map (previous section). From these thermal maps, a temporal temperature gradient is derived by comparison on a pixel-by-pixel basis to detect changes in the rooftop temperature patterns that might be related to heat-loss fluxes. The temperature gradient $(\Delta \mathrm{T})$ is segmented as very low, low, medium or high if $\Delta \mathrm{T}<\mathrm{T}_{\text {mean }}-\sigma_{\Delta \mathrm{T}}$, $\mathrm{T}_{\text {mean }}-\sigma_{\Delta \mathrm{T}}<\Delta \mathrm{T}<\mathrm{T}_{\text {mean }}, \quad \mathrm{T}_{\text {mean }}<\Delta \mathrm{T}<\mathrm{T}_{\text {mean }}+\sigma_{\Delta \mathrm{T}}$ and $\mathrm{T}_{\text {mean }}+\sigma_{\Delta \mathrm{T}}<\Delta \mathrm{T}$, respectively. Note that the segmentation thresholds are fixed after examining the overall temperature gradient on the roofs 
in the area of interest, where Tmean is the average temperature gradient on the rooftops and $\sigma \Delta \mathrm{T}$ is the standard deviation of the temperature gradient. Finally, a file is derived for each industrial building footprint summarizing the information on the temperature gradient (see figure 7). This file, in pdf format, contains a full-res detail of the night thermal map, the early morning thermal map, temperature gradient on the rooftop, orthoimage, temperature gradient segmentation and the legend. Further explanations may be found in (Pipia et al. 2014b).
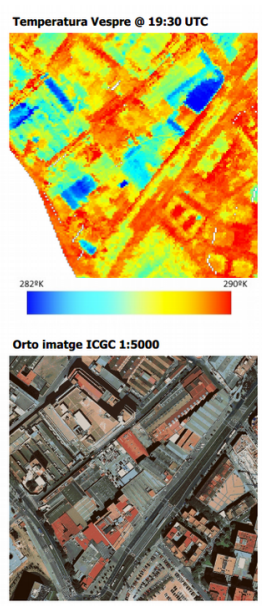
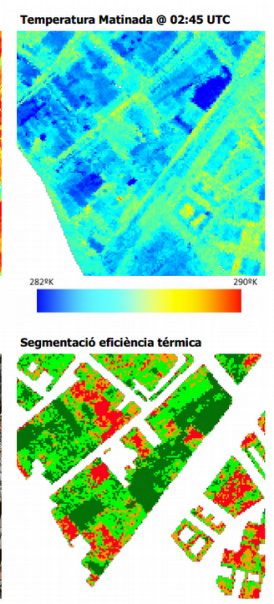
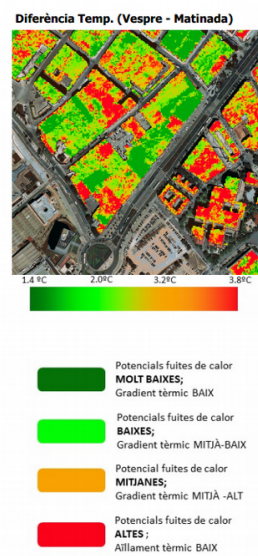

Figure 7. Top left: thermal image at night. top centre: thermal image early in the morning, top right: temperature gradient at rooftop, bottom left: orthoimage, bottom centre: temperature gradient segmentation and bottom right: legend.

\subsection{Solar potential}

The UHI map is derived from the data acquired during the close-to-midnight.

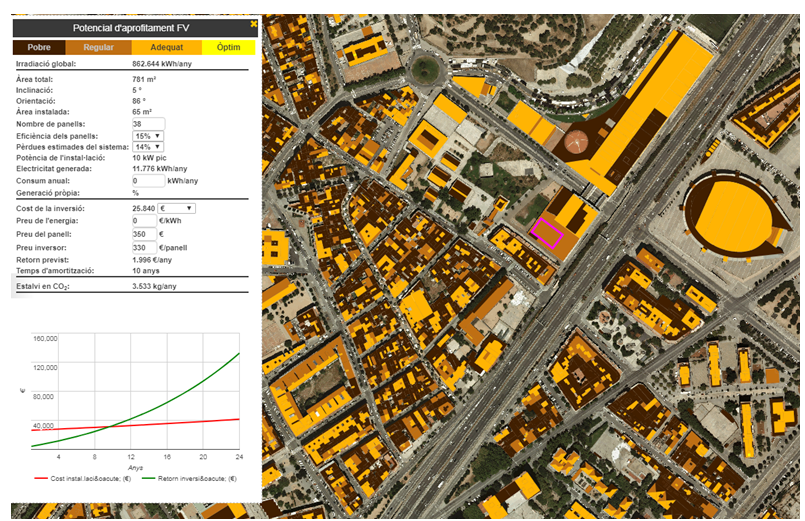

Figure 8. Suitability index for the deployment of photovoltaic energy facilities

A $0.5 \mathrm{~m}$ GSD DSM is derived from the Lidar point cloud. For each building footprint, a pixel-by-pixel seasonal shading analysis is performed taken into account the DSM, which contains information on buildings, vegetation and topography, by running simulations (one day per week, 18 hours per day) along the whole year. These data are combined to produce a global and diffuse irradiation map for the whole year on the building rooftops. This accumulated sunlight map takes into account the local weather conditions (clouds) and airmass effects (heze, pollution). These effects have been modelled through monthly atmosphere transparency coefficients for the sun direct and diffuse radiation, in turn derived from long series of observations in different meteorological stations.
Rooftop planes are detected from a building LoD2 model, which has been derived from the DSM. For each rooftop plane, suitability indices for the deployment of photovoltaic (FV) and solar thermal (ST) energy facilities are computed (see figures 8 and 9 , respectively). These suitability indices are segmented into poor, medium, fine and optimum for $\mathrm{FV}$ and poor fine and optimum for ST (see Magariños and Ruiz 2017).

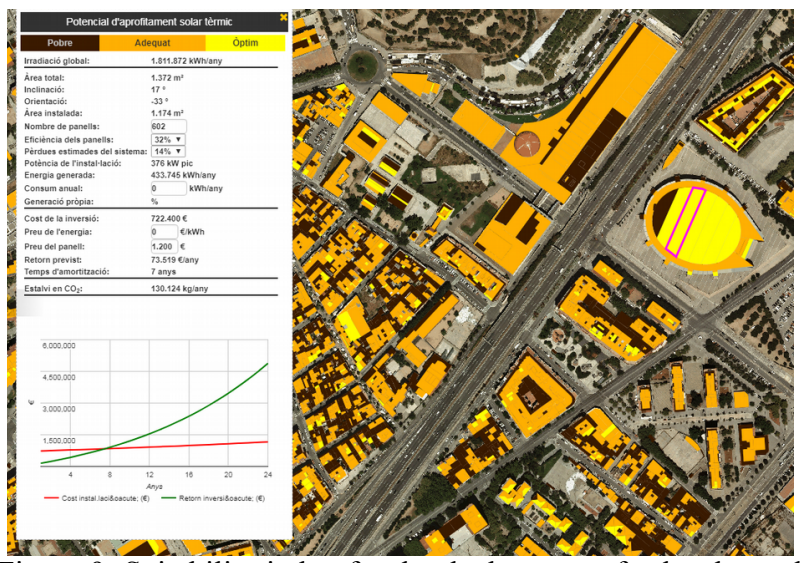

Figure 9. Suitability index for the deployment of solar thermal energy facilities

\subsection{Urban Green}

The urban green map is derived from the data acquired at diurnal flights with a standard photogrammetric camera.

The photogrammetric camera captures four multispectral wide bands in the VisNIR: near-infrared, red, green and blue. Thus, there is the capability to derive the well-known NDVI index.

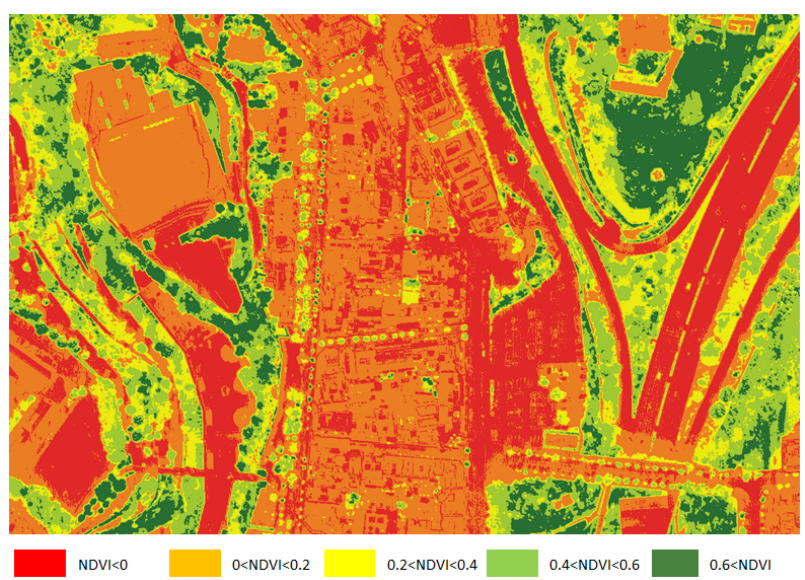

Figure 10. Urban green (NDVI) map

A standard aerial triangulation of the set of images is performed in order to obtain the orientation of the images. From the images and using image matching techniques a DSM of the threes is derived. Then, an orthoimage is generated by mosaicking all the images and the urban green (NDVI) map at $40 \mathrm{~cm}$ GSD is derived. The NDVI is segmented in 5 classes $\mathrm{NDVI}<0$ (water or man-made surfaces), $0<\mathrm{NDVI}<0.2$ (natural ground with no vegetation), $0.2<\mathrm{NDVI}<0.4 \quad$ (sparse vegetation), $0.4<\mathrm{NDVI}<0.6$ (vegetation) and NDVI $>0.6$ (dense and healthy vegetation) (see figure 10). 


\section{CONCLUSIONS}

The operational approach of the ICGC is providing a set of maps and urban indicators covering a wide spectrum of urban features.

Firstly, ICGC is providing high resolution luminance maps (up $25 \mathrm{~cm}$ GSD) and light source typology maps (up $1.5 \mathrm{~m}$ GSD). As these maps are carried out in a single flight session they provide a synoptic view and thematic information of the nocturnal urban landscape. These maps become a tool to analyse and monitor both public and private illumination, check whether policies on light pollution are fulfilled (in terms of intensity or typology of light sources) and evaluate street lighting policies and actuations.

Secondly, ICGC is providing thermal maps up to $2 \mathrm{~m}$ GSD resolution. From them, UHI maps and temperature gradient maps on rooftops are derived. Such maps provide a synoptic view of the thermal behaviour of the municipalities. These maps, by themselves or correlated with additional socioeconomic data, are a tool to develop policy and planning guides to limit UHI intensities, to detect potential heat-loss fluxes on infrastructures or buildings and to promote policies and actuations to improve building thermal isolation.

Thirdly, ICGC is providing suitability maps for the deployment of solar energy facilities: photovoltaic and solar thermal energy installations. These maps are a tool to promote policies and actuations on renewable energy.

Finally, ICGC is providing urban green maps, which provide a synoptic view of the urban vegetation landscape. These maps become a tool to analyse vegetation distribution and vigour, both in public and private domains and, linking them with other data as UHI maps and/or socioeconomic data, promote informed policies and actuations.

Summarizing, ICGC is providing a set of maps and urban indicators over the urban ecosystem. There are two features to highlight: i) datasets are acquired in a short period of time (a single flight most of the times), which is very important for the analysis of parameters or indicators that change quickly in time as temperature, and ii) the different sensors involved from standard photogrammetric cameras to hyperspectral sensors in VisNIR and LWIR. Such dataset is a tool to support and develop urban planning policies and energy policies at municipality level.

\section{ACKNOWLEDGEMENTS}

The authors would like to thank the Diputació de Barcelona and the municipal administration of Badalona as stakeholders that demanded ICGC products and their fruitful comments on correct interpretation of the retrieved information.

\section{REFERENCES}

Alamús, R., Palà, V., Tardà, A., Pipia, L. and Corbera, J., 2017. COperational light source and luminance determination methodology by using hyperspectral and mapping cameras at very high spatial resolution. In: The 5th International Symposium on Recent Advances in Quantitative Remote Sensing: RAQRS'V.

Barsi, J.B., Barker J.L. and Schott J.R., 2003. An Atmospheric Correction Parameter Calculator for a Single Thermal Band Earth-Sensing Instrument. In: Geoscience and Remote Sensing
Symposium 2003, IGARSS'03 Proceedings, Vol 5, pp. 30143016.

\author{
CIE, 1931. CIE Comission Internationale de l'Eclairage \\ Proceedings. Cambridge University Press
}

Dörstel, C., 2003. DMC- Practical Experiences and Photogrammetric System Performace. In: Proceedings of the 49th PhoWo, pp. 59-65.

Harris, R., Coutts, A., 2011. Technical Report Airborne Thermal Remote Sensing for Analysis of the Urban Heat Island http://www.vcccar.org.au/publication/technicalreport/airborne-thermal-remote-sensing-urban-heat-island (last access 13 July 2018).

Magariños, A., Ruiz, A., 2017. Estudi del potencial solar a Rubí. In: Revista Catalana de Geografia IV època / volum XXII / núm. 55. http://www.rcg.cat/articles.php?id=388 (last access 13 July 2018)

Pipia, L., Alamús, R., Tardà, A., Pérez F., Palà, V., Corbera, J., 2014. A methodology for luminance map retrieval using airborne hyperspectral and photogrammetric data. In: Proc. SPIE 9245, Earth Resources and Environmental Remote Sensing/GIS Applications V,92450O.

Pipia, L., Pérez, F., Tardà, A., Corbera, J., Morera, M., Ruiz, A., 2014b. Hyperspectral TIR sensor for building heatloss detection. In: The 4th International Symposium on Recent Advances in Quantitative Remote Sensing: RAQRS'IV.

Pipia, L., Pérez, F., Tardà, A., Martínez, L., Palà, V., Arbiol, R., 2013. Thermal Airborne Spectrographic Imager for Temperature and Emissivity Retrieval. In: The 3rd International Symposium on Recent Advances in Quantitative Remote Sensing: RAQRS'III. 